\title{
ABSOLUTE SUMMABILITY MATRICES THAT ARE STRONGER THAN THE IDENTITY MAPPING
}

\author{
J. A. FRIDY
}

ABSTRACT. The main result gives a simple column-sum property which implies that the matrix $A$ maps $l_{A}$ properly into $l^{1}$, i.e., $l^{1} \varsubsetneqq A^{-1}\left[l^{1}\right]$. Also, the means of Nörlund, Euler-Knopp, Taylor, and Hausdorff are investigated as mappings of $l^{1}$ into itself.

1. Introduction. Let $A$ be an infinite matrix defining a sequence to sequence summability transformation by $(A x)_{n}=\Sigma_{k \geq 1} a_{n k}{ }_{k}$. The inverse image of $l^{1}$ under $A$ is denoted by $l_{A}$; and $A$ is called an $l-l$ matrix provided that $l^{1} \subseteq l_{A}$. In [7] Knopp and Lorentz proved that $A$ is an $l-l$ matrix if and only if there is a number $M$ such that for each $k$,

$$
\sum_{n \geq 1}\left|a_{n k}\right| \leq M
$$

Also, $A$ is sum-preserving if for each $x$ in $l^{1}, \Sigma_{n \geq 1}(A x)_{n}=\Sigma_{k \geq 1} x_{k}$. The $l-l$ matrix $A$ is sum-preserving if and only if for each $k$,

$$
\sum_{n \geq 1} a_{n k}=1
$$

In [2] Agnew gave a simple sufficient condition that $A$ maps a nonconvergent sequence into a convergent one. The principal result of this paper is an analogue of this condition for $l-l$ matrices; i.e., we shall give an explicit property of the terms $\left\{a_{n k}\right\}$ that implies $l \varsubsetneqq l_{A}$. In the final section, we investigate the absolute summability properties of some well-known matrix methods.

2. The main theorem. Following Agnew, we might conjecture that $\lim _{n, k} a_{n k}=0$ implies $l_{A} \neq l$. (The double limit is taken in the Pringsheim

Received by the editors November 12, 1973.

AMS (MOS) subject classifications (1970). Primary 40C05, 40D05, 40D25, 40F 05, 40G05; Sec ondary 40D15.

Key words and phrases. l-l matrix, sum-preserving, Nörlund means, Euler-Knopp means, Taylor method, Hausdorff means, quasi-Hausdorff means, regular mass function. 
sense: $\left|a_{n k}\right|<\epsilon$ whenever $n>N$ and $k>N_{\text {. }}$ ) This conjecture is reinforced by the observation that it is true in case $A$ is lower triangular with $a_{n n} \neq 0$; for then $A^{-1}$ is not $l-l$ because $\sup _{n}\left|a_{n n}\right|^{-1}=\infty$. However, the following example shows that, even for lower triangular matrices, this property is not sufficient in general.

Example. If

$$
a_{n k}= \begin{cases}1 / k, & \text { if } k(k-1) / 2<n \leq k(k+1) / 2, \\ 0, & \text { otherwise, }\end{cases}
$$

then $A$ is a lower triangular, sum-preserving $l-l$ matrix for which $\lim _{n, k} a_{n k}$ $=0$, but $l_{A}=l$.

If Agnew's property is replaced by $\lim _{k} \Sigma_{n \geq 1}\left|a_{n k}\right|=0$, then it is easy to construct an unbounded sequence $x$ such that $\Sigma_{n}\left|(A x)_{n}\right|$ converges. Indeed, it is sufficient that only a subsequence of the column sums tends to zero, so we can state the following result (cf. [4 Proposition]).

Proposition. If $A$ is a matrix such that

$$
\underset{k}{\lim \inf } \sum_{n \geq 1}\left|a_{n k}\right|=0
$$

then $l_{A} \neq l$.

The simplicity of condition (3) is offset by the fact that it precludes (2), and therefore, no sum-preserving matrix can satisfy it. It is, therefore, necessary to weaken (3), which leads us to the main result.

Theorem 1. If $A$ is an $l-l$ matrix for which there exists an integer $m$ such that

$$
\underset{k}{\lim \inf } \sum_{n \geq m}\left|a_{n k}\right|=0
$$

then $l \varsubsetneqq l_{A}$.

Proof. The hypothesis (4) implies the existence of an increas ing integer sequence $\{k(i)\}$ such that for each $i, \Sigma_{n \geq m}\left|a_{n, k(i)}\right|<1 / i$. If $x$ is chosen so that $\left|x_{k(i)}\right| \leq 1 / i$, and $x_{k}=0$ when $k \neq k(i)$, then

$$
\begin{aligned}
\sum_{n \geq m}\left|(A x)_{n}\right| & \leq \sum_{n \geq m} \sum_{i \geq 1}\left|a_{n ; k(i)}\right| i^{-1} \\
& =\sum_{i \geq 1} \sum_{n \geq m}\left|a_{n, k(i)}\right| i^{-1}<\sum_{i \geq 1} i^{-2} .
\end{aligned}
$$


Hence, $A x$ is in $l^{1}$ provided that $x$ is in the domain of $A$. The difficulty is that we must ensure the convergence of $\Sigma_{i} a_{n, k(i)} x_{k(i)}$ when $n<m$. To achieve this we must choose a subsequence $\{\kappa(i)\}$ of $\{k(i)\}$ so that for each $n<m, \Sigma_{i} a_{n, \kappa(i)} x_{\kappa(i)}$ converges, while $\left|x_{\kappa(i)}\right|=1 / i$. Since (1) implies that the row sequences are bounded, the proof will be completed by the following lemma.

Lemma. If, for each $n$ less than $m,\left\{a_{n k}\right\}_{k=1}^{\infty}$ is a bounded sequence, then there exists a sequence $x$ that is not in $l^{1}$ such that $\Sigma_{k} a_{n k} x_{k}$ converges for each $n$ less than $m$.

Proof. Let $M_{n}$ denote $\lim \sup _{k}\left|a_{n k}\right|$, and assume-without loss of generality-that $M_{1} \geq M_{2} \geq \cdots \geq M_{m-1} \geq 0$. We may also assume that the $a_{n k}$ 's are real, for otherwise we could treat $\left\{\operatorname{Re}\left(a_{n k}\right)\right\}_{k=1}^{\infty}$ and $\left\{\operatorname{Im}\left(a_{n k}\right)\right\}_{k=1}^{\infty}$ separately and have $2(m-1)$ bounded sequences.

First, suppose $M_{m-1}>0$. Choose a subsequence $\left\{a_{1, k(i)}\right\}_{i}$ of $\left\{a_{1, k}\right\}_{k}$ such that the terms are either all positive or all negative and for each $i$,

$$
\frac{i+1}{i+2} M_{1} \leq\left|a_{1, k(i)}\right| \leq \frac{i^{2}}{i^{2}-1} M_{1} .
$$

Now choose a subsequence $\left\{k^{*}(i)\right\}$ of $\{k(i)\}$ so that all of the terms $\left\{a_{2, k^{*}(i)}\right\}$ are of the same sign and for each $i$,

$$
\frac{i+1}{i+2} M_{2} \leq\left|a_{2, k^{*}(i)}\right| \leq \frac{i^{2}}{i^{2}-1} M_{2} .
$$

Choose successive subsequences so that after $m-1$ selections, we have a subsequence $\{\kappa(i)\}$ of the positive integers such that if $n<m$, then $\left\{a_{n, \kappa(i)}\right\}_{i}$ are all of the same sign, and for each $i$,

$$
\frac{i+1}{i+2} M_{n} \leq\left|a_{n, K(i)}\right| \leq \frac{i^{2}}{i^{2}-1} M_{n} .
$$

Now define $x$ by

$$
x_{k}= \begin{cases}(-1)^{i} / \cdot i, & \text { if } k=\kappa(i) \text { for some } i, \\ 0, & \text { otherwise. }\end{cases}
$$

Then $\Sigma_{k} a_{n k} x_{k}=\Sigma_{i} a_{n, k(i)}(-1)^{i} / i$. This is obviously an alternating series whose general term tends to 0 . Also, from (5) we have

$$
\frac{1}{i}\left|a_{n, \kappa(i)}\right| \leq \frac{i}{i^{2}-1} M_{n} \leq \frac{1}{i-1} \frac{i}{i+1} M_{n-1} \leq \frac{1}{i-1}\left|a_{n, \kappa(i)}\right| .
$$


Hence, by the familiar a lternating series test, $\Sigma_{k} a_{n k} x_{k}$ is convergent.

In case $M_{m-1}=0$, the preceding construction will yield an $x$ for which $\Sigma_{k} a_{n k} x_{k}$ converges when $M_{n}>0$. Then remaining sequences $\left\{a_{n k}\right\}$ for which $M_{n}=0$ are null sequences, and the selection of subsequences for which $\Sigma_{k} a_{n k} x_{k}$ converges is straightforward. Hence, $\Sigma_{k} a_{n k} x_{k}$ converges for every $n$ less than $m$, which completes the proof of the Lemma and the Theorem.

Although it is possible for a sum-preserving $l-l$ matrix to satisfy (4), it is easy to see that no lower triangular matrix can satisfy both (2) and (4). Indeed, if $A$ is lower triangular, then (4) implies (3). This leads us to conjecture that perhaps a weaker condition, such as $\lim \inf _{k}\left|\Sigma_{n \geq m} a_{n k}\right|=0$, might be sufficient to imply $l_{A} \neq l^{1}$. However, if

$$
a_{n k}=\left\{\begin{aligned}
1, & \text { if } n=2 k-1 \\
-1, & \text { if } n=2 k \\
0, & \text { otherwise, }
\end{aligned}\right.
$$

then $\Sigma_{n \geq m} a_{n k}=0$ if $k \neq m / 2$, but clearly $l_{A}=l$.

3. Absolute summability properties of some classical methods. Since many of the classical means are given by lower triangular matrices with nonzero diagonal terms, we can use the following observation in place of (3) for such matrices: $l_{A} \varsubsetneqq l^{1}$ if and only if $A$ satisfies (1) but $A^{-1}$ does not. Note that (3) implies that $A^{-1}$ does not satisfy (1) because $\sup _{n}\left|a_{n n}\right|^{-1}=\infty$.

The Nörlund mean $N_{p}$ is given by $N_{p}[n, k]=p_{n-k} / P_{n}$ if $k \leq n$, and $N_{p}[n, k]=0$ if $k>n$, where $p$ is a nonnegative number sequence such that $p_{0}>0$ and $P_{n} \equiv \Sigma_{k=0}^{n} p_{k}$. in $l^{1}$.

Theorem 2. The Nörlund mean $N_{p}$ is an l-l matrix if and only if $p$ is

Proof. If $p$ is in $l^{1}$, then for each $k$

$$
\sum_{n=0}^{\infty} N_{p}[n, k]=\sum_{n=k}^{\infty} \frac{p_{n-k}}{P_{n}} \leq P_{0}^{-1} \sum_{n=k}^{\infty} p_{n-k}=p_{0}^{-1} \sum_{i=0}^{\infty} p_{i} ;
$$

hence, $N_{p}$ satisfies (1). Conversely, if $p$ is not in $l^{1}$, then by a result of Abel (see footnote in [6, p. 45]), $\lim _{n}\left\{1 / P_{n}\right\}=0$ while $\Sigma_{n} p_{n} / P_{n}$ diverges. Thus $N_{p}$ is not an $l-l$ matrix.

From Theorem 2, we see that if $l^{1} \subseteq l_{N_{p}}$, then $\lim \inf _{k}\left\{p_{k} / P_{k}\right\}>0$, so property (4) does not hold. However, we can prove a Merceriantype theorem. 
For, if $1 / 2<r<1$ and $p_{0} \geq r P_{n}$, then $N_{p}[n, n] \geq r$ (for every $n$ ). Therefore, by $\left[5\right.$, Theorem 6], we conclude that $l_{N_{p}}=l^{1}$. This proves the following as. sertion.

Theorem 3. If $p_{0}>2 \Sigma_{k \geq 1} p_{k}$, then $l_{N_{p}}=l^{1}$.

A particular example of Theorem 3 is seen in case $p$ is a geometric sequence: more precisely, if $p_{k+1}<p_{0} 3^{-k-1}$, then $l_{N_{p}}=l^{1}$.

The Euler-Knopp means ([1], [6], and [9]) are given by

$$
E_{r}[n, k]= \begin{cases}\left(\begin{array}{l}
n \\
k
\end{array}\right) r^{k}(1-r)^{n-k}, & \text { if } k \leq n, \\
0, & \text { if } k>n .\end{cases}
$$

A straightforward application of the Maclaurin series expansion of $(1-z)^{k+1}$ shows that each column sum of $E_{r}$ converges absolutely to $1 / r$ provided that $0<r \leq 1$. If $0<r<1$, then $\lim _{n} E_{r}[n, n]=0$, so $E_{r}^{-1}$ is not an $l-l$ matrix. We summarize this as follows:

Theorem 4. The Euler-Knopp mean $r E_{r}$ is a sum-preserving l-l matrix for which $l_{E_{r}} \neq l^{1}$ if and only if $0<r<1$.

The Taylor methods ([3], [8], and [9]), which are given by

$$
T_{r}[n, k]= \begin{cases}0, & \text { if } k<n, \\
\left(\begin{array}{l}
n \\
k
\end{array}\right) r^{k-n}(1-r)^{n+1}, & \text { if } k \geq n,\end{cases}
$$

are related to the Euler-Knopp means by a transpose relationship. More precisely, if $E^{*}$ denotes the transpose of $E$, then $T_{r}=(1-r) E_{1-r}^{*}$. It follows that $T_{r}$ is an $l-l$ matrix for precisely those $r$ 's for which $E_{r}$ maps bounded sequences into bounded sequences, viz., $0 \leq r \leq 1$. We note that (4) is not satisfied by $T_{r}$, when $0 \leq r<1$; for, each column sum is $1-r$, and since the first $m$ rows are null sequences we must have $\Sigma_{n \geq m} a_{n k} \geq(1-r) / 2$ for sufficiently large $k$.

The Hausdorff means ([6] and [9]) can be defined by

$$
H_{\phi}[n, k]=\int_{0}^{t} E_{t}[n, k] d \phi(t),
$$

where $E_{t}$ is the Euler-Knopp mean and $\int_{0}^{1}|d \phi|<\infty$. The quasi-Hausdorff mean $H_{\phi}^{*}$ is simply the transpose of $H_{\phi}$. Therefore, $H_{\phi}$ is an $l-l$ method if and only if $H_{\phi}^{*}$ is a bounded operator, and Hardy [6, pp. 278-279] characterizes this by $\int_{0}^{1}|d \phi(t)| / t<\infty$. Furthermore, the column sums are 


$$
\sum_{n=0}^{\infty} H_{\phi}[n, k]=\sum_{k=0}^{\infty} H_{\phi}^{*}[n, k]=\int_{0}^{1} \frac{d \phi(t)}{t} .
$$

Thus we may state the following result.

Theorem 5. The Hausdorff matrix $H_{\phi}$ generated by the mass function $\phi$ is an $l-l$ matrix if and only if $\int_{0}^{1}|d \phi(t)| t^{-1}$ converges. Moreover, $H_{\phi}$ is sum-preserving if and only if $\int_{0}^{1} t^{-1} d \phi(t)=1$.

The corresponding theorem for $H_{\phi}^{*}$ can be stated without proof since it depends upon only the regularity conditions for $H_{\phi}$ [6, pp. 256-258].

Theorem 6. The quasi-Hausdorff matrix $H_{\phi}^{*}$ is an $l-l$ method if and only if $\phi$ is a function of bounded variation on $[0,1]$. Moreover, $H_{\phi}^{*}$ is sumpreserving if and only if $\phi(1)-\phi(0)=1$.

Note that in Theorem 6 it is not required that $\phi\left(0_{+}\right)=\phi(0)$, so $\phi$ need not be a "regular" mass function. Since $\phi\left(0_{+}\right)-\phi(0)=\lim _{n} H_{\phi}[n, 0]=$ $\lim _{k} H_{\phi}^{*}[0, k]$, it might seem possible that $H_{\phi}^{*}$ is an $l-l$ method and satisfies (4). Unfortunately this cannot be the case, because if $k>0$, then

$$
\lim _{n} H_{\phi}[n, k]=0 \text {, }
$$

and

$$
\begin{aligned}
\sum_{n \geq m}\left|H_{\phi}^{*}[n, k]\right| & =\sum_{n=0}^{\infty}\left|H_{\phi}^{*}[n, k]\right|-\sum_{n=0}^{m-1}\left|H_{\phi}^{*}[n, \dot{k}]\right| \\
& =\int_{0}^{1}|d \phi|-\left|H_{\phi}^{*}[0, k]\right|-\sum_{n=1}^{m-1}\left|H_{\phi}^{*}[n, k]\right| \\
& =\frac{1}{2} \int_{0+}^{1}|d \phi|
\end{aligned}
$$

for $k$ sufficiently large.

Finally, we remark on the conspicuous absence from our study of the very familiar Cesàro means. The fact is that they are not $l-l$ methods. For, if $\alpha>0$ and $\phi(t)=1-(1-t)^{\alpha}$, then $H_{\phi}$ is the Cesàro mean of order $\alpha[6$, p. 275]. But clearly $\int_{0}^{1} t^{-1} \cdot d \phi(t)$ is divergent, so by Theorem $5, C_{a}$ is not an $l-l$ method.

\section{REFERENCES}

1. R. P. Agnew, Euler transformations, Amer. J. Math. 66 (1944), 313-338. MR 6, 46 . 
2. R. P. Agnew, A simple sufficient condition that a method of summability be stronger than convergence, Bull. Amer. Math. Soc. 52 (1946), 128-132. MR 7, 292.

3. V. F. Cowling, Summability and analytic continuation, Proc. Amer. Math. Soc. 1 (1950), 536-542. MR 12, 91.

4. J. A. Fridy, Properties of absolute summability matrices, Proc. Amer. Math. Soc. 24 (1970), 583-585. MR $42 \# 724$.

5. - Mercerian-type theorems for absolute summability, Portugal. Math. 33 (1974), 141-145.

6. G. H. Hardy, Divergent series, Clarendon Press, Oxford, 1949. MR 11, 25.

7. K. Knopp and G. G. Lorentz, Beiträge zur absoluten Limitierung, Arch. Math. 2 (1949), 10-16. MR 11, 346.

8. W. Meyer-König, Untersuchungen über einige verwandte Limitierungsverfahren, Math. Z. 52 (1949), 257-304. MR 11, 242.

9. R. E. Powell and S. M. Shah, Summability theory and applications, Van Nostrand Reinhold, London, 1972.

DEPARTMENT OF MATHEMATICS, KENT STATE UNIVERSITY, KENT, OHIO 44242 